\title{
Exploration to the effect of psychological nursing on the negative emotion of patients with skin disease
}

\author{
Zhang Mengsi \\ Jingzhou Institute of Technology Hubei Jingzhou 434020
}

Keywords: skin disease patients; psychological nursing; negative emotion; rehabilitation effect; influence

\begin{abstract}
China's social economy, the change of people's lifestyle and cultural level, the cognitive level of the disease is also constantly improving. For most patients with skin diseases, due to the long-term and repetitive characteristics of their own diseases, it is relatively difficult during the actual treatment period, which leads to the patients are prone to anxiety, panic etc. Poor psychological emotions such as helplessness seriously affect the rehabilitation process. Based on this, this study carried out around patients with skin diseases, aiming to analyze the clinical effect of psychological nursing intervention measures, hoping to provide reference for relevant practitioners.
\end{abstract}

\section{Introduction}

Skin, as the largest organ of human body, is also its first physiological defense line. It always participates in various functional activities of the body. It can effectively reflect the health status of the body and maintain the unity of opposites with the natural environment. Moreover, the skin also has many physiological protection functions, such as regulating body temperature, barrier function, absorption function, excretion function etc. It will cause the appearance of skin diseases, and the types of diseases are relatively more, such as psoriasis, scar, herpes, psoriasis, whelk, folliculitis, etc., and such patients will also have different degrees of rash, measles, rubella, chickenpox, pruritus symptoms at the onset of the disease, which will have a serious impact on the physical and mental health of patients ${ }^{[1]}$. At present, for this kind of disease, clinicians often take drug treatment measures. However, during this period, some skin disease patients are prone to varying degrees of psychological disorders, coupled with the prognosis of the worry, and then seriously affect its final efficacy. Therefore, timely and effective psychological care for patients with skin disease is particularly important ${ }^{[2]}$.

\section{Induced etiology}

According to modern medicine, the causes of skin diseases include: (1) physical and chemical factors. Such as: pressure and friction, local temperature changes too fast, light, heat radiation, chemical reagents, etc., at the same time, on the basis of the skin disease, excessive scratching, washing with soap and water or improper medication will aggravate the degree of skin lesions; (2) biological factors. Including: mosquito bites, plant pollen allergy, parasites, viruses and microbial infections ${ }^{[3]}$. (3) Food or other disease irritation. Fish and shrimp allergy or local infection, blood, lymph circulation disorders, etc., are easy to induce infectious eczema like dermatitis and cyanosis. (4) Metabolic and endocrine factors, such as skin amyloidosis and xanthoma. 5) Family inheritance. Such as, the ichthyosis and albinism.

\section{Psychological nursing}

\subsection{Psychological comfort}

After patients with skin diseases are admitted to hospital, clinical staff must inquire about the patient's name, age, basic condition and other information at the first time, formulate the 
corresponding electronic medical record file, and introduce the specific environment of the hospital, the location of each department and the corresponding rules and regulations for skin disease patients, so as to eliminate the fear of the unknown environment in patients ${ }^{[4]}$. At the same time, nursing staff also need to actively communicate with patients with skin diseases, encourage patients to bravely express their inner thoughts, for patients put forward reasonable needs, nursing staff can also try to meet; and in the actual treatment process, nursing staff also need to consider for patients from multiple angles, with the help of patients' interests to divert their attention, so as to achieve improvement The role of adverse emotions in patients with skin diseases ${ }^{[5]}$.

\subsection{Health education}

In the process of receiving treatment, some skin disease patients are prone to anxiety, fear, helplessness and other negative psychology due to the lack of understanding of disease-related knowledge and the long-term development of various adverse symptoms of the disease. Therefore, nursing staff can issue disease brochures for them, organize and carry out corresponding health knowledge lectures, in order to popularize the knowledge Easy to understand language for patients to explain the causes of skin disease, clinical manifestations, treatment programs, etc., correct the patient's concept of disease; at the same time, for some patients with relatively low education level and more serious inferiority complex, nursing staff can also invite successful cases in our hospital for one-to-one communication to help patients better understand the appearance of their own skin diseases Knowledge, as soon as possible to adapt to the role of patients, improve their cognitive level and treatment compliance, enhance the patient's self-confidence ${ }^{[6] \text {. }}$

\subsection{Relaxation training}

Nursing staff can also encourage patients to participate in corresponding entertainment items in the activity room of the ward in their spare time, such as playing cards, playing chess, reading books, video games, etc., so as to better divert the attention of patients and release their heavy psychological pressure; at the same time, for some patients with relatively serious illness, nursing staff can also guide them They were trained in deep breathing, progressive muscle relaxation and imaginative relaxation, and each exercise lasted for 15-20 minutes, once a day ${ }^{[7]}$.

\subsection{Family support}

In the process of hospitalized patients with skin disease, nursing staff also need to strengthen the communication and communication with the patients' family members, instruct the family members to give them more care and accompany as far as possible, and inform the patients that there is no need to worry about the cost of treatment, so as to better eliminate the loneliness of skin patients and provide a strong psychological support for patients.

\subsection{Environmental guidance}

The head nurse of the Department shall arrange special personnel to clean, clean and disinfect the ward where the patients with skin disease are located, timely open windows for ventilation, ensure the circulation of fresh air, properly adjust the indoor temperature, humidity and light conditions, strictly control the number of people visiting relatives and friends, so as to create a comfortable treatment environment for skin disease patients, ensure their good sleep quality and promote their heart The improvement of emotional pleasure ${ }^{[8]}$. In addition, for some patients with infectious skin diseases, nursing staff also need to arrange a special treatment area for them, so as to reduce the activity in the public area as much as possible, and avoid the malignant spread of the disease.

In conclusion, in the process of skin disease patients receiving treatment, clinical related staff can take psychological nursing intervention measures, so as to better ensure the sleep quality of patients, improve their anxiety, depression and other negative emotions, and improve the treatment compliance of patients. 


\section{References}

[1] Rabiba gesbaywa. Analysis of psychological status and nursing care of patients with allergic skin diseases [J]. Diet and health care, 2018, 005 (029): 183-184

[2] Weng Liyan, Wu Weilian. Effect of individualized psychological intervention on anxiety and depression of psoriasis patients in Luoding City [J]. Chinese medical innovation, 2018, 015 (011): $70-73$

[3] Wang Metxia. Effect of intensive psychological nursing on psychological state and immune cytokines in patients with chronic urticaria [J]. Dermatology and venereal diseases, 2019, 41 (02): 286-288

[4] Li Fang, Ma Xingyun. Effect analysis of psychological nursing combined with health education in the nursing of burn and plastic surgery patients [J]. Dermatology and venereal diseases, 2018, 40 (06): 151-152

[5] Zhang Chunhua, Huang Yongmei, Wang Xiaoyan, et al. Effect analysis of comprehensive nursing intervention on sensitive skin diseases in plateau area [J]. China continuing medical education, 2019, 11 (11): 205-206

[6] Dai Xiaomei. Analysis of the effect of positive psychological nursing on improving psychological status and behavior of patients with psoriasis [J]. Modern diagnosis and treatment, 2018, 029 (021): 3559-3560

[7] Shen Yanyan, Li Huali. Application and effect of holistic nursing in children with skin diseases [J]. Dermatology and venereal diseases, 2019, 041 (003): 451-453

[8] Zhou Ji. Effect of integrated traditional Chinese and Western medicine combined with continuous nursing on the quality of life of patients with chronic urticaria [J]. Dermatology and venereal diseases, 2018, 040 (005): 688-689 